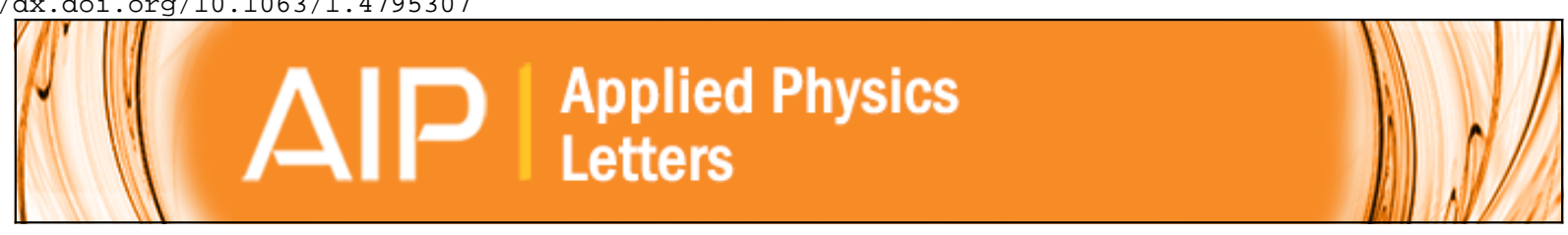

Nonlinear magnetoelectric response of a Metglas/piezofiber laminate to a highfrequency bipolar AC magnetic field

Yaojin Wang, Ying Shen, Junqi Gao, Menghui Li, Jiefang Li, and D. Viehland

Citation: Applied Physics Letters 102, 102905 (2013); doi: 10.1063/1.4795307

View online: http://dx.doi.org/10.1063/1.4795307

View Table of Contents: http://scitation.aip.org/content/aip/journal/apl/102/10?ver=pdfcov

Published by the AIP Publishing

AlP Re-register for Table of Content Alerts 


\title{
Nonlinear magnetoelectric response of a Metglas/piezofiber laminate to a high-frequency bipolar AC magnetic field
}

\author{
Yaojin Wang (汪尧进), ${ }^{\text {a) }}$ Ying Shen, Junqi Gao, Menghui Li, Jiefang Li, and D. Viehland \\ Materials Science and Engineering, Virginia Tech, Blacksburg, Virginia 24061, USA
}

(Received 13 February 2013; accepted 28 February 2013; published online 13 March 2013)

\begin{abstract}
A nonlinear magnetoelectric (ME) response has been investigated in a Metglas/piezofiber laminate by applying a bipolar AC magnetic field $\left(H_{\mathrm{ac}}\right)$ without a DC magnetic bias. The ME voltage $\left(V_{\mathrm{ME}}\right)$ was measured for various amplitudes of $H_{\mathrm{ac}}$ of up to 9 Oe over the frequency $(f)$ range $0.1<f<40 \mathrm{kHz}$. Compared to the linear ME behavior, an anomalous response to $f$ and $H_{\mathrm{ac}}$ was observed, which is believed to be associated with the Eddy-current loss in the Metglas layers. A frequency multiplication effect was also observed for $V_{\mathrm{ME}}$, which was understood by Fourier analysis of the nonlinearity in the magnetostriction. (C) 2013 American Institute of Physics.

[http://dx.doi.org/10.1063/1.4795307]
\end{abstract}

Magnetoelectric (ME) composites, which consist of magnetostrictive and piezoelectric components, are very attractive in comparison with single-phase ME materials owing to their technologically viable ME coefficient. ${ }^{1,2}$ In such composites, the ME effect is a product property of the magnetostrictive and piezoelectric effects of the corresponding phases and their coupling. In previous reports, motivated by passive magnetic sensor applications, a linear response of ME composites to a weak AC magnetic field $H_{\mathrm{ac}}$ was theoretically and experimentally developed for various composite constitutions, configurations, and operation modalities. ${ }^{3-5}$ A milestone in the development of ME composites was the appearance of ones operated in multi-push-pull mode, consisting of magnetostrictive Metglas foils and piezoelectric $\mathrm{Pb}\left(\mathrm{Mg}_{1 / 3} \mathrm{Nb}_{2 / 3}\right) \mathrm{O}_{3^{-}}$ $\mathrm{PbTiO}_{3}(\mathrm{PMN}-\mathrm{PT})$ crystal or $\mathrm{Pb}\left(\mathrm{Zr}_{\mathrm{x}} \mathrm{Ti}_{1-\mathrm{x}}\right) \mathrm{O}_{3}(\mathrm{PZT})$ ceramic fibers with interdigitated (ID)-electrodes. ${ }^{6}$ The ME characterization for passive detection in such laminate composites involves a measurement of an induced ME voltage $\left(V_{\mathrm{ME}}\right)$ across the ID-electrodes in response to a harmonic incident field of amplitude $0<H_{\mathrm{ac}}<0.1 \mathrm{Oe}$ and of frequency $0<f<100 \mathrm{kHz}$ under various DC magnetic biases of $-30<H_{\mathrm{dc}}<30$ Oe. ${ }^{6,7}$

Recently, ME laminate composites have also been employed as active detection elements to sense a small DC magnetic field ${ }^{8,9}$ or weak AC magnetic field, via a modulation-demodulation technique. ${ }^{10}$ A bipolar $H_{\mathrm{ac}}$ with a relative high amplitude of several Oersted is needed to drive the ME laminate. An induced signal $V_{\mathrm{ME}}$ represents the amplitude of a small DC magnetic field or a weak AC magnetic field that is to be detected. However, there have only been a few investigations of the nonlinear ME effect of laminate composites in response to a bipolar magnetic excitation to date. $^{11}$

Here, the ME effect in a heterostructure consisting of magnetostrictive Metglas layers, piezoelectric single crystal fibers, and a pair of ID-electrodes was investigated by subjecting the laminate to a time varying bipolar AC magnetic field $H_{\text {ac }}$. In particular, a nonlinear response to $H_{\mathrm{ac}}$ was observed at various frequencies, which was caused by corresponding

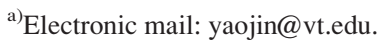

nonlinearities in the magnetostriction. A frequency multiplication effect was also observed for $V_{\mathrm{ME}}$, which was related to a Fourier analysis of the nonlinear magnetostriction.

A multi-pull-pull configuration laminate comprised of Metglas foils, Mn-doped PMN-PT crystal fibers, and a pair of ID-electrodes with a center-to-center spacing of $1.5 \mathrm{~mm}$ was employed for this investigation. The fabrication details for this type of sensor can be found in previous reports. ${ }^{6,12,13}$ First, the magnetostriction was characterized using a straingauge method [see Figure 1(a)], the linear ME coefficient as a function of $H_{\mathrm{dc}}$ at constant amplitude of $H_{\mathrm{ac}}=0.5 \mathrm{Oe}$ at $f=1 \mathrm{kHz}$ [see Figure 1(b)], and the linear ME coefficient as a function of frequency at $H_{\mathrm{dc}}=8 \mathrm{Oe}$ and $H_{\mathrm{ac}}=0.05 \mathrm{Oe}$. The piezomagnetic coefficient $(\mathrm{d} \lambda / \mathrm{d} H)$, calculated directly from the slope of the $\lambda-H_{\mathrm{dc}}$ curves, is also presented in Fig. 1(a), which shows a peak value of $\sim 2.2 \mathrm{ppm} \mathrm{Oe}^{-1}$ at $H_{\mathrm{dc}}=8 \mathrm{Oe}$. In addition, the magnetostriction was well fit by a five-order Fourier polynomial over the measurement range of $H$, as will be discussed later. The variation of the ME coefficient $\alpha_{\mathrm{V}}$ with $H_{\mathrm{dc}}$ was functionally similar to that of the effective linear piezomagnetic coefficient: this is because $\alpha_{\mathrm{V}}$ is directly proportional to the piezomagnetic coefficient. ${ }^{6}$ Figure 1(c) shows the dependence of $\alpha_{\mathrm{V}}$ on frequency over the range of $0.1<f<40 \mathrm{kHz}$ under the optimal $H_{\mathrm{dc}}$. No significant dispersion of $\alpha_{\mathrm{V}}$ was observed, apart from the variations associated with the longitudinal-extensional electromechanical resonance (EMR), where a giant ME coefficient of over $115 \mathrm{~V} / \mathrm{Oe}$ was obtained.

The nonlinear ME voltage coefficient was then measured as a function of frequency over the range of $0.1<f<40 \mathrm{kHz}$ at various amplitude of $H_{\mathrm{ac}}$ under zero bias field, as shown in Figure 2(a). The bipolar $H_{\mathrm{ac}}$ was provided by a lab-built Helmholtz coil serially combining with a $10 \mathrm{k} \Omega$ resistor, which was driven by a lock-in amplifier (SR785) coupled with a power amplifier (Trek Model 609D-6). It can be seen that $V_{\mathrm{ME}}$ increased with increase of $H_{\mathrm{ac}}$ in both the sub-resonance and resonance ranges, as more clearly shown in the inset of this figure. It can also be seen that $V_{\mathrm{ME}}$ notably decreased with increase of $f$ away from the resonance frequency. Figure 2(b) shows $V_{\mathrm{ME}}$ as a function of $H_{\mathrm{ac}}$ at frequencies of $f_{\mathrm{ac}}=1 \mathrm{kHz}$, $10 \mathrm{kHz}, 20 \mathrm{kHz}$, and EMR. A nonlinear response to $H_{\mathrm{ac}}$ is 

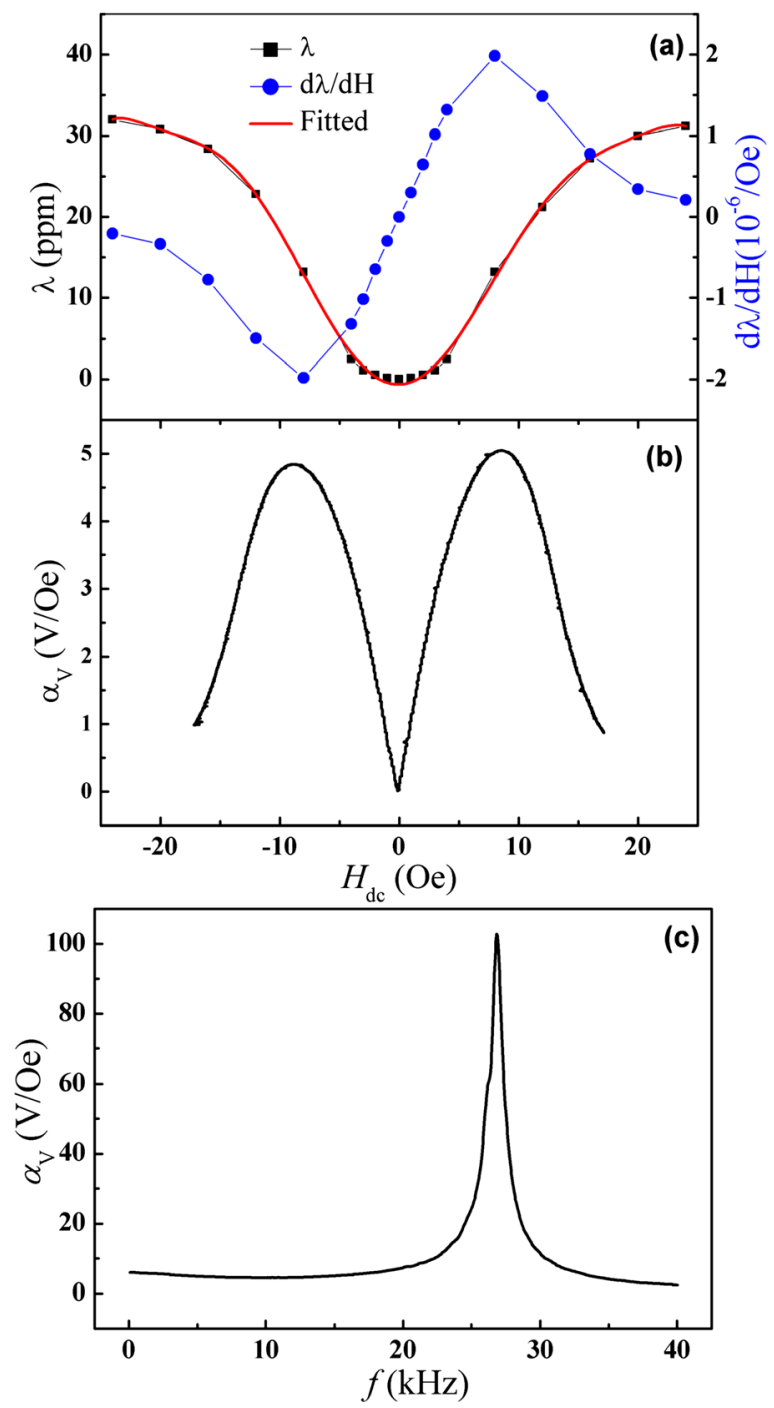

FIG. 1. The DC magnetic bias $H_{\mathrm{dc}}$ dependence of (a) the longitudinal magnetostriction $\lambda$ and effective linear piezomagnetic coefficient, and (b) the linear ME voltage coefficient of a Metglas/piezofiber laminate sensor. A Fourier polynomial was used to fit the $\lambda-H_{\mathrm{dc}}$ curve. (c) The ME coefficient as a function of frequency over the range of $0.1<f<40 \mathrm{kHz}$ under the optimal $H_{\mathrm{dc}}=8$ Oe.

obvious. Unlike the linear effect, the nonlinear $V_{\mathrm{ME}}$ at $1 \mathrm{kHz}$ was notably higher than that at $20 \mathrm{kHz}$; an enhancement factor of about 5 can be seen near resonance. This anomalous characteristic may be associated with a dynamic magnetization process, such as domain wall motion.

In soft magnetic materials, such as Metglas foils, Eddycurrent induced losses dominate the total energy dissipation: i.e., the hysteresis losses of the domain wall motion are negligible. The Kelvin ber and bei functions can be used to predict the frequency dependence of the real and imaginary portions of the permeability, ${ }^{14}$ given as

$$
\begin{gathered}
\frac{\mu_{\Re e}^{\text {theory }}}{\mu^{\text {zero }}}=\frac{2}{\theta} \frac{\operatorname{ber} \theta \operatorname{bei}^{\prime} \theta-\operatorname{bei} \theta \operatorname{ber}^{\prime} \theta}{\operatorname{ber}^{2} \theta+\operatorname{bei}^{2} \theta}, \\
\frac{\mu_{\Im m}^{\text {theory }}}{\mu^{\text {zero }}}=\frac{2}{\theta} \frac{\operatorname{ber} \theta \operatorname{bei}^{\prime} \theta+\operatorname{bei} \theta \operatorname{ber}^{\prime} \theta}{\operatorname{ber}^{2} \theta+\operatorname{bei}^{2} \theta},
\end{gathered}
$$

where
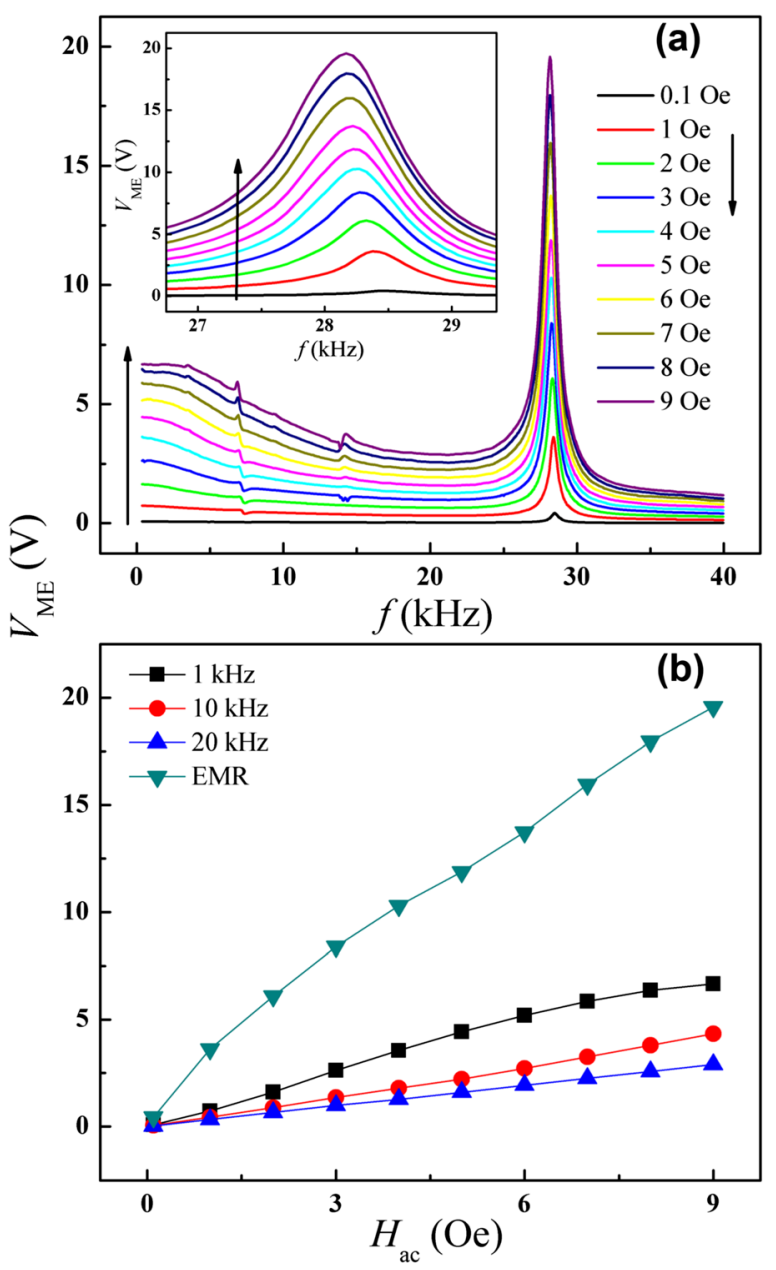

FIG. 2. (a) The induced ME voltage $V_{\mathrm{ME}}$ as a function of frequency over the range of $0.1<f<40 \mathrm{kHz}$ under various amplitudes of $H_{\mathrm{ac}}$. The inset shows an enlarged view near the EMR condition. (b) The dependence of $V_{\mathrm{ME}}$ on the amplitude of $H_{\mathrm{ac}}$ at frequencies of $f_{\mathrm{ac}}=1,10,20 \mathrm{kHz}$, and the EMR condition.

$$
\theta=\left(\frac{2 S \mu^{z e r o}}{\rho_{R}} f\right)^{1 / 2}
$$

and where $\mu^{\text {zero }}$ is the dynamic permeability extrapolated to zero frequency, and $\rho_{\mathrm{R}}$ and $S$ are the resistivity and crosssectional area of the Metglas foils. The Eddy-current loss $\left(W_{\text {ec }}\right)$ can then be given by ${ }^{15}$

$$
W_{e c} \equiv \pi H_{p k}^{2} \mu_{0} \mu^{\text {zero }}\left(\frac{\mu_{\Im m}^{\text {theory }}}{\mu^{\text {zero }}}\right)=\frac{\pi S\left(\mu_{0} \mu^{\text {zero }} H_{p k}\right)^{2} f}{4 \rho_{R}} ;
$$

where $H_{\mathrm{pk}}$ is the peak value of the applied bipolar magnetic field. From Eq. (4), it can be seen as the frequency increases that the Eddy-current induced energy loss becomes larger. This leads to a reduced dynamic magnetostriction, in turn resulting in a decrease in $V_{\mathrm{ME}}$ with increase of $f_{\mathrm{ac}}$ over the sub-resonance frequency range, as shown in Fig. 2.

Figures 3(a)-3(c) show time domain waveforms of the induced $V_{\mathrm{ME}}$ in response to $H_{\mathrm{ac}}$ for $f=1 \mathrm{kHz}$. The amplitude of $H_{\mathrm{ac}}$ was varied from $0.1 \mathrm{Oe}$ to $5 \mathrm{Oe}$, and the data for $V_{\mathrm{ME}}$ were recorded by an oscilloscope (Agilent 54624A). A time domain waveform was observed that was atypical of that of linear ME effect: ${ }^{12,16}$ this is because a bipolar $H_{\mathrm{ac}}$, even at a low amplitude of $0.1 \mathrm{Oe}$, results in a departure of $V_{\mathrm{ME}}$ from a 

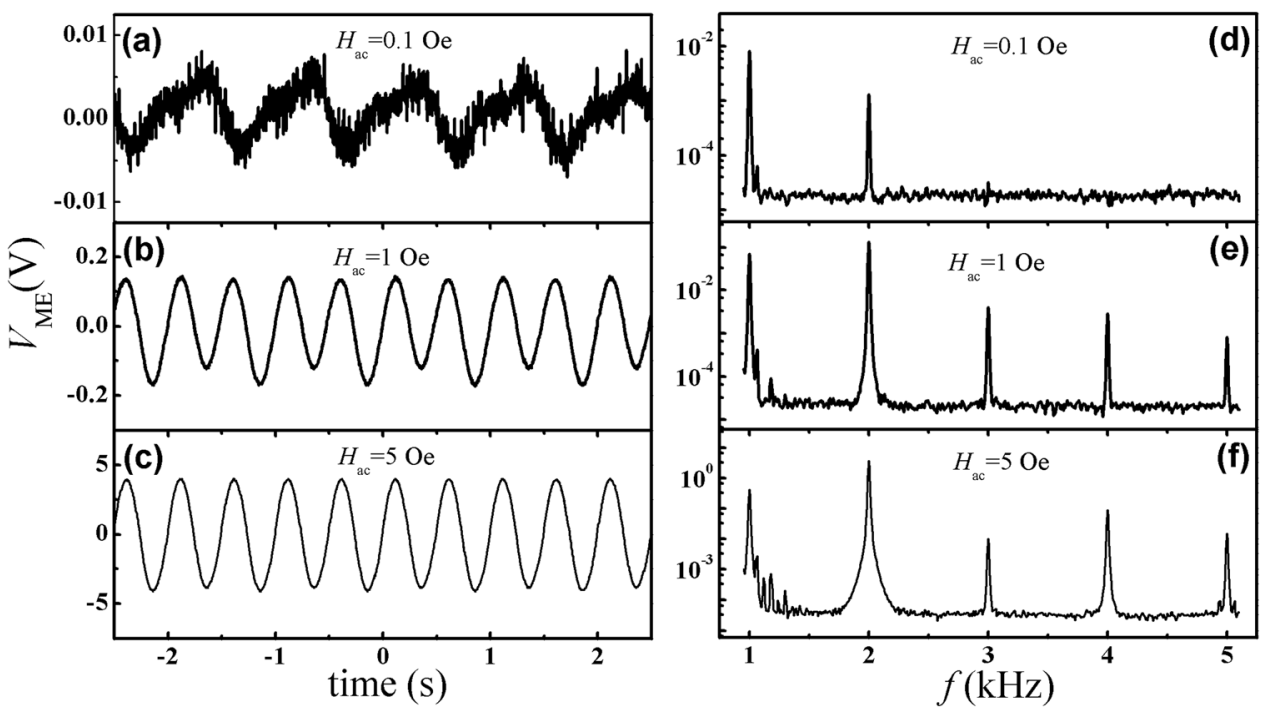

FIG. 3. The measured $V_{\mathrm{ME}}$ as a time domain waveform for (a) $H_{\mathrm{ac}}=0.1 \mathrm{Oe}$, (b) $H_{\mathrm{ac}}=1 \mathrm{Oe}$, (c) $H_{\mathrm{ac}}=5 \mathrm{Oe}$ and as a FFT spectral pattern for (d) $H_{\mathrm{ac}}=0.1 \mathrm{Oe}$, (e) $H_{\mathrm{ac}}=1 \mathrm{Oe}$, and (f) $H_{\mathrm{ac}}=5 \mathrm{Oe}$. pure sinusoidal variation. Furthermore, for $H_{\mathrm{ac}} \geq 5 \mathrm{Oe}$, a further distortion of the $V_{\mathrm{ME}}$ profile can be seen that resulted in frequency-doubling. In order to more accurately characterize these atypical responses, a FFT frequency spectral pattern for $V_{\mathrm{ME}}$ was measured using a Dynamic Signal Analyzer (SR850), as shown in Figs. 3(d)-3(f). In addition to a peak response to $H_{\mathrm{ac}}$ at $f_{\mathrm{ac}}=1 \mathrm{kHz}$, several harmonic peaks were observed at $f=2,3,4$, and $5 \mathrm{kHz}$ over the frequency range of $0.9<f<5.5 \mathrm{kHz}$, which coincided with previous reports. ${ }^{11,17}$ Please note that the relative amplitude of the harmonic peaks increased with the increase of $H_{\mathrm{ac}}$.

This harmonic ME effect can be explained by a nonlinearity in magnetostriction (see Fig. 1(a)). The magnetostriction $\lambda$ of the ME laminate can be given as a five-order Fourier expansion series, given as

$$
\lambda=a_{0}+a_{1} H+a_{2} H^{2}+a_{3} H^{3}+a_{4} H^{4}+a_{5} H^{5}
$$

where $H=H_{\mathrm{ac}} \cos \left(2 \pi f_{\mathrm{ac}} t\right)$ is the applied magnetic field, and $a_{i}(i=0,1,2 \ldots)$ are $i$ th Fourier coefficients. The value of $a_{\mathrm{i}}$ was independent of $H_{\mathrm{ac}}$ and could simply be determined by fitting Eq. (5) to the $\lambda-H$ data of Fig. 1(a): where $a_{0}=-6.4 \times 10^{-7}, a_{1}=3.7 \times 10^{-8} / \mathrm{Oe}, a_{2}=2.6 \times 10^{-7} / \mathrm{Oe}^{2}$, $a_{3}=-1.4 \times 10^{-9} / \mathrm{Oe}^{3}, a_{4}=-8.9 \times 10^{-10} / \mathrm{Oe}^{4}$, and $a_{5}=7.4$ $\times 10^{-12} / \mathrm{Oe}^{5}$. Then, Eq. (5) can be rewritten as

$$
\begin{aligned}
\lambda= & A_{0}+A_{1} \cos \left(2 \pi f_{a c} t\right)+A_{2} \cos \left(4 \pi f_{a c} t\right) \\
& +A_{3} \cos \left(6 \pi f_{a c} t\right)+A_{4} \cos \left(8 \pi f_{a c} t\right)+A_{5} \cos \left(10 \pi f_{a c} t\right)
\end{aligned}
$$

where $A_{0}=a_{0}+\frac{1}{2} a_{2} H_{a c}^{2}+\frac{3}{8} a_{4} H_{a c}^{2}, \quad A_{1}=a_{1} H_{a c}+\frac{3}{4} a_{3} H_{a c}^{3}$ $+\frac{5}{8} a_{5} H_{a c}^{5}, \quad A_{2}=\frac{1}{2} a_{2} H_{a c}^{2}+\frac{1}{2} a_{4} H_{a c}^{4}, A_{3}=\frac{1}{4} a_{3} H_{a c}^{3}+\frac{5}{16} a_{5} H_{a c}^{5}$, $A_{4}=\frac{1}{8} a_{4} H_{a c}^{4}$ and $A_{5}=\frac{5}{16} a_{5} H_{a c}^{5}$.

The rearranged Fourier coefficients of Eq. (6) are determined not only by the initial Fourier coefficients $a_{\mathrm{i}}$, but are also dependent on $H_{\mathrm{ac}}$. This accounts for the change in the relative amplitude of the harmonic peaks with $H_{\mathrm{ac}}$ (see Figs. 3(d)-3(f)). It is worth noting that in addition to the harmonic peaks in Fig. 3(a), a voltage peak at $f_{\text {ac }}=0$ was found that was due to a DC strain component related to $A_{0}{ }^{11,17}$
Figure 4 shows the measured $V_{\mathrm{ME}}$ and the calculated Fourier coefficient $A_{\mathrm{i}}$ (or predicted $V_{\mathrm{ME}}$ ) for the different orders of harmonics. The measured values were directly obtained from Fig. 3(b), and the predicted ones from $A_{\mathrm{i}}$ at various amplitudes of $H_{\mathrm{ac}}$. In Fig. 4, it can be seen that for all cases the variations in harmonics of the induced $V_{\mathrm{ME}}$ were similar to those in the Fourier coefficients $A_{\mathrm{i}}$. This gives strong support to the conjecture that the nonlinear $\mathrm{ME}$ response of the laminates is directly proportional to the magnetostriction of the Metglas foils. It also can be seen that the measured $V_{\mathrm{ME}}$ and calculated Fourier $A_{\mathrm{i}}$ at the 1st-order harmonic were both notably higher than that at higher-order harmonics at a low amplitude of $H_{\mathrm{ac}}=0.1$; whereas, under higher excitations of $H_{\mathrm{ac}}=1$ and $5 \mathrm{Oe}$, the 2nd-order harmonic was the strongest.

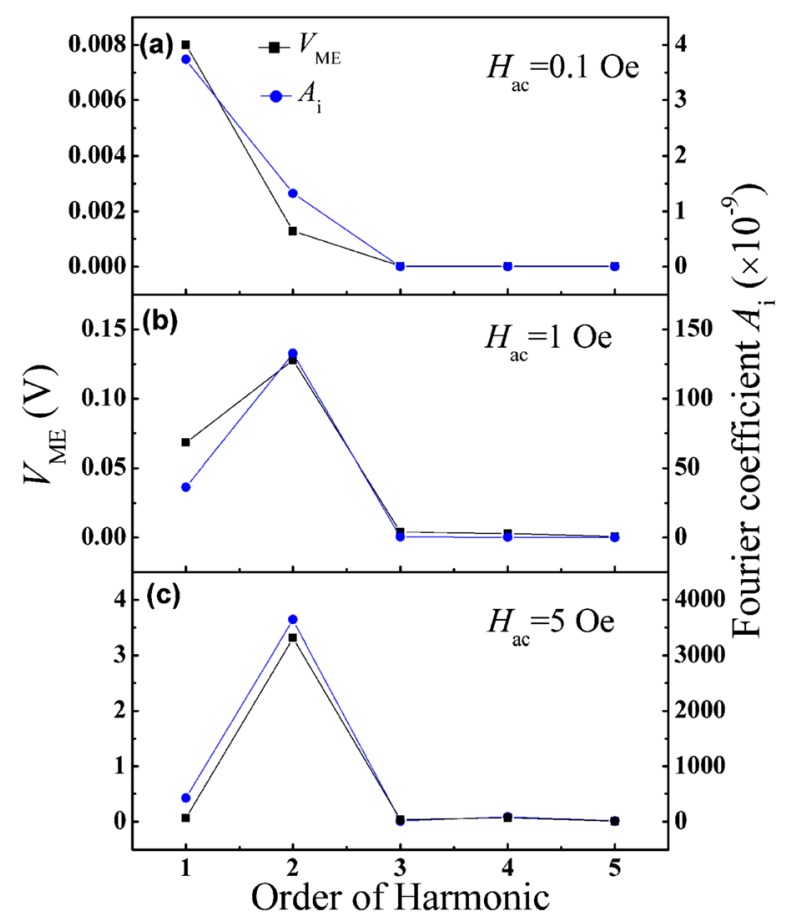

FIG. 4. Measured $V_{\mathrm{ME}}$ and calculated Fourier coefficients $A_{\mathrm{i}}$ for various harmonics at (a) $H_{\mathrm{ac}}=0.1 \mathrm{Oe}$, (b) $H_{\mathrm{ac}}=1 \mathrm{Oe}$, and (c) $H_{\mathrm{ac}}=5 \mathrm{Oe}$. 
In summary, the nonlinear ME effect in Metglas/piezofiber heterostructures has been investigated. Measurements were performed over wide frequency $(0.1-40 \mathrm{kHz})$ and drive (0.1-9 Oe) ranges. It was found that the nonlinear $V_{\mathrm{ME}}$ coefficient increased with increase of $H_{\mathrm{ac}}$ and decreased with increase of $f$ except at EMR. A nonlinearity in the magnetostriction of the Metglas layers results in frequency multiplication and strong higher-order harmonics.

This work was sponsored by the Office of Naval Research (N00014-3-1-0128).

${ }^{1}$ E. Lage, C. Kirchhof, V. Hrkac, L. Kienle, R. Jahns, R. Knoechel, E. Quandt, and D. Meyners, Nature Mater. 11, 523 (2012).

${ }^{2}$ J. Jin, S.-G. Lu, C. Chanthad, Q. Zhang, M. A. Hague, and Q. Wang, Adv. Mater. 23, 3853 (2011).

${ }^{3}$ M. I. Bichurin, V. M. Petrov, S. V. Averkin, and E. Liverts, J. Appl. Phys. 107, 053904 (2010).

${ }^{4}$ C. W. Nan, M. I. Bichurin, S. X. Dong, D. Viehland, and G. Srinivasan, J. Appl. Phys. 103, 031101 (2008).
${ }^{5}$ D. Hasanyan, J. Gao, Y. Wang, R. Viswan, M. Li, Y. Shen, J. Li, and D. Viehland, J. Appl. Phys. 112, 013908 (2012).

${ }^{6}$ Y. J. Wang, D. Gray, D. Berry, J. Q. Gao, M. H. Li, J. F. Li, and D. Viehland, Adv. Mater. 23, 4111 (2011).

${ }^{7}$ S. X. Dong, J. Y. Zhai, J. F. Li, and D. Viehland, Appl. Phys. Lett. 89, 252904 (2006).

${ }^{8}$ S. X. Dong, J. Y. Zhai, J. F. Li, and D. Viehland, Appl. Phys. Lett. 88, 082907 (2006).

${ }^{9}$ J. Gao, D. Gray, Y. Shen, J. Li, and D. Viehland, Appl. Phys. Lett. 99, 153502 (2011).

${ }^{10}$ R. Jahns, H. Greve, E. Woltermann, E. Quandt, and R. Knoechel, Sens. Actuators A 183, 16 (2012).

${ }^{11}$ K. E. Kamentsev, Y. K. Fetisov, and G. Srinivasana, Appl. Phys. Lett. 89, 142510 (2006).

${ }^{12}$ Y. Wang, D. Gray, D. Berry, M. Li, J. Gao, J. Li, and D. Viehland, J. Alloys Compd. 513, 242 (2012).

${ }^{13}$ Y. Wang, J. Gao, M. Li, D. Hasanyan, Y. Shen, J. Li, D. Viehland, and H. Luo, Appl. Phys. Lett. 101, 022903 (2012).

${ }^{14}$ R. M. Bozorth, Ferromagnetism (IEEE Press, Piscataway, NJ, 1993).

${ }^{15}$ D. Kendall and A. R. Piercy, J. Appl. Phys.73, 6174 (1993).

${ }^{16}$ Y. J. Wang, S. W. Or, H. L. W. Chan, X. Y. Zhao, and H. S. Luo, Appl. Phys. Lett. 93, 213504 (2008).

${ }^{17}$ W. Zhang, G. Yin, J. Cao, J. Bai, and F. Wei, Appl. Phys. Lett. 100, 032903 (2012). 